\title{
Imaging at the Single-Atom Level in Closed-Cell In Situ Gas Reactions
}

\author{
Lawrence F. Allard ${ }^{1}$, Sibin Duan ${ }^{2}$ and Jingyue (Jimmy) Liu ${ }^{2}$ \\ 1. Materials Science \& Technology Div., Oak Ridge National Laboratory, Oak Ridge, TN 37831, USA. \\ 2. Department of Physics, Arizona State University, Tempe, Arizona 85287, USA.
}

Single-atom catalysis is emerging as a new frontier in the field of heterogeneous catalysis [1-4]. Singleatom catalysts (SACs) have recently been successfully synthesized and proved to be effective for many important catalytic reactions with high atom efficiency [1-4]. A key issue facing the practical applications of SACs is, however, the stability of the metal single atoms during catalytic reactions, especially at elevated temperatures. To develop robust SAC synthesis protocols to stabilize noble metal single atoms on appropriate metal oxide supports requires a fundamental understanding of the dynamics of the supported single atoms under gas environments and at elevated temperatures. Understanding the dynamical movement and the anchoring of noble metal atoms on support materials becomes critical to developing novel strategies to synthesize SACs with high levels of metal loading. Recently developed technologies for in situ reaction studies utilizing a closed-cell MEMS-based reactor specimen holder [5] in an aberration-corrected (S)TEM instrument have shown the capability to image atomic columns on metal particles at pressures up to an atmosphere and temperatures up to $1000^{\circ} \mathrm{C}$. However, imaging of single heavy-metal atoms on oxide supports and tracking their movements during reaction experiments remains problematical, due to the scattering phenomena relative to the elements of the gas cell geometry, and electron beam effects. In the present study, we demonstrate the capabilities and efficacy for observations of single-atom reactions in a closed-cell system, using a specimen comprising Pt atoms on $\mathrm{NiO}$ nanocrystals. High-angle annular dark-field (HAADF) mages are shown for 4 cases: (a) the ideal geometry of catalyst support particles suspended over a hole in a holey-carbon support film; (b) support particles on the carbon film; (c) support particles on the thin (30nm) SiN membrane of a MEMS heater device with the beam first traversing the $\mathrm{SiN}$ (see caption for details); and (d) support particles imaged in the full gas-cell geometry at elevated temperature and pressure (see caption).

Figure 1a shows case (a), HAADF images of $\mathrm{Pt}$ atoms on $\mathrm{NiO}$ over a hole so no additional noise in the image from the vacuum background. Figure $1 \mathrm{~b}$ shows the $\mathrm{NiO}$ support over the $\mathrm{C}$ film, illustrating little to no additional noise background in HAADF mode due to the lack of scattering at high angles by the $10 \mathrm{~nm} \mathrm{C}$ film. Figure 2 is a schematic of the typical MEMS reactor geometry: a heater device is the upper element, and the support particles sit on the lower surface of an amorphous SiN membrane suspended over holes in a ceramic heater membrane. Gold spacers provide a nominal $5 \mathrm{~nm}$ gas path for the scattered electron to pass through, and a second $\operatorname{SiN}(50 \mathrm{~nm})$ "window" membrane serves to contain the gas, and is the final element through which scattered electrons pass to finally reach the HAADF and $\mathrm{BF}$ detectors. Figure $3 \mathrm{a}$ illustrates case (c), and shows that the electron beam passing through the $\mathrm{SiN}$ layer on the heater element does not significantly degrade the image resolution or contrast, as the light $\mathrm{SiN}$ film does not provide strong scattering into the HAADF detector and therefore does not add strongly to the background noise in the image. Under a typical set of gas-cell operating parameters, as shown in Figure $3 \mathrm{~b}\left(50\right.$ Torr flowing $\left.\mathrm{O}_{2}, 300^{\circ} \mathrm{C}\right)$, single atoms are still visible on the $\mathrm{NiO}$ surface, with crystal lattice also seen in the $\mathrm{NiO}$ particle, illustrating the promise to track the behavior of single-atom catalysts under a series of reaction environments, and ultimately the potential to obtain valuable information on mechanisms of reactions catalyzed by single-atom species, an important new thrust in catalytic science [6]. 


\section{References:}

[1] Q. Fu, H. Saltsburg, M. Flytzani-Stephanopoulos, Science 301 (2003), p. 935.

[2] B. Qiao et al., Nat. Chem. 3 (2011), p. 634.

[3] X. Yang et al., Acc. Chem. Res. 46 (2013), p. 1740.

[4] M. Yang et al., J. Am. Chem. Soc. 135 (2013), p. 3768.

[5] L. F. Allard, et al., Micros. Microanal. 18 (2012), p. 656.

[6] Microscopy research at ORNL sponsored in part by the U.S. Dept. of Energy (DOE), Office of Energy Efficiency and Renewable Energy, Vehicle Technologies Program, as part of the Propulsion Materials Program, and (JL and SD) by the National Science Foundation under CHE-1465057 and Arizona State University.
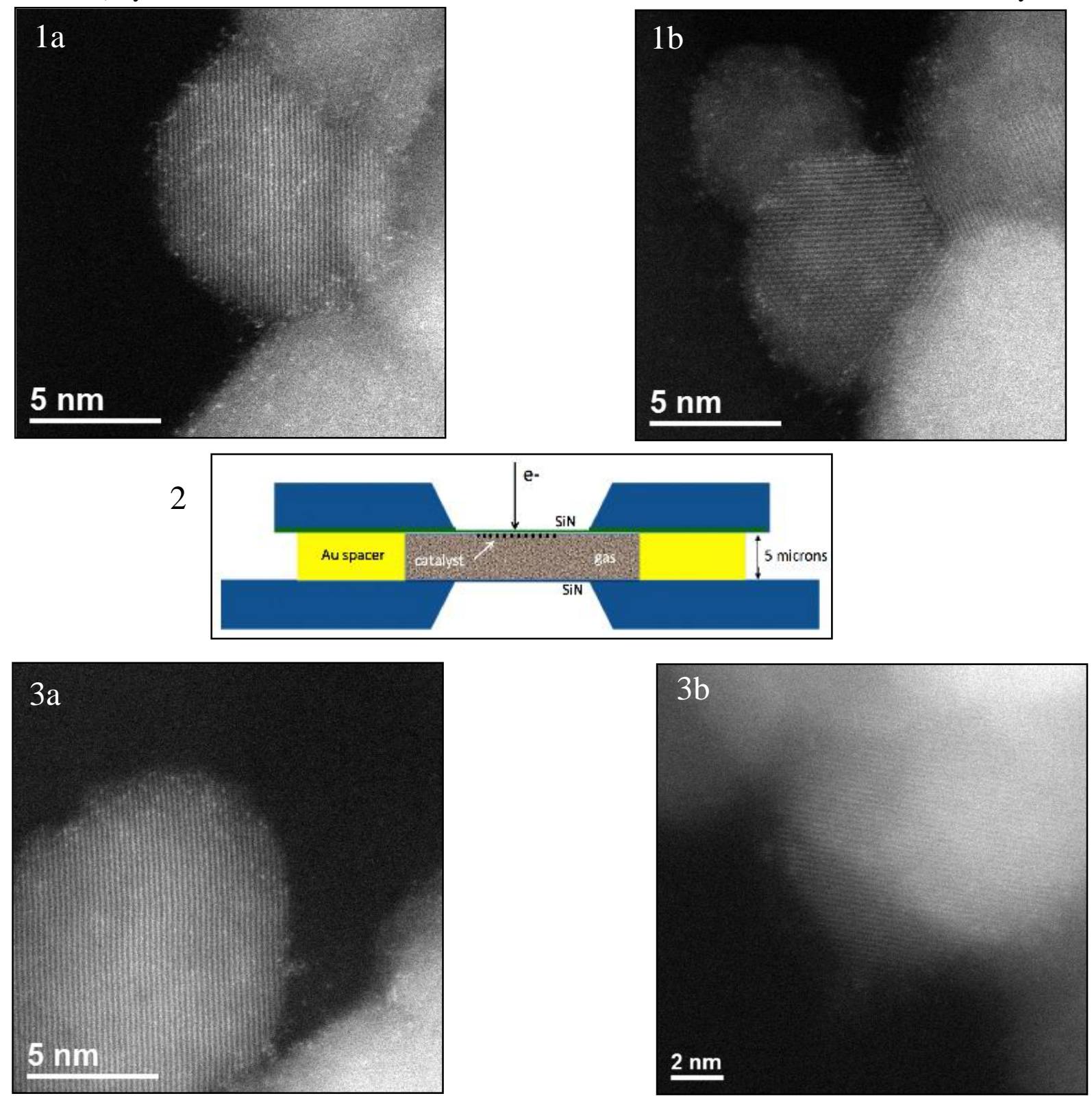

Figure 1. Pt atoms on $\mathrm{NiO}$ imaged (a) with $\mathrm{NiO}$ over hole to give vacuum background; (b) over C-film, showing no contrast in HAADF due to C-film scattering. Figure 2. Schematic of MEMS heater, with upper element SiN heater.

Figure 3. (a) Pt atoms imaged with the beam through the SiN layer; and (b) in full gas-cell geometry. 